grade osteosarcoma arising from FD in the femur. ${ }^{5}$ Immunohistochemically, this FD was negative for p53 and MDM2, whereas the low grade osteosarcomatous component was positive for both p53 and MDM2. ${ }^{5}$ Both studies, including ours, demonstrated TP53 mutations. TP53 mutation was central to the diagnosis of osteosarcoma, which would have been difficult on morphology alone, especially so in small biopsies. This was important as neoadjuvant chemotherapy is the standard of care for osteosarcoma as it has been shown to improve event free survival and overall survival. $^{11}$

Other factors that limited the diagnosis of osteosarcoma in small biopsies were small sample size, not being representative of the entire lesion and sampling underdeveloped atypia instead. In our case, TP53 mutation was detected in the small biopsies taken, which was used to support the diagnosis of osteosarcoma.

In summary, this is a rare report of high grade osteosarcoma arising from FD of the maxilla supported by GNAS and TP53 mutation analysis. Our findings suggest that GNAS and TP53 mutations can be used to support osteosarcomatous transformation in fibrous dysplasia.

Conflicts of interest and sources of funding: The authors state that there are no conflicts of interest to disclose.

Francis H. X. Yap ${ }^{1}$, Benhur Amanuel ${ }^{1}$, Chris Van Vliet ${ }^{1}$, Marc Thomas ${ }^{1}$, Daniel Wong ${ }^{1,2}$

${ }^{1}$ Department of Anatomical Pathology, PathWest, QEII Medical Centre, Nedlands, WA, Australia; ${ }^{2}$ School of Pathology and Laboratory Medicine, The University of Western Australia, Crawley, WA, Australia

Contact Dr Francis H. X. Yap.

E-mail: Hong.yap@ health.wa.gov.au

1. Fletcher CD, Bridge JA, Hogendoorn PCW, et al. WHO Classification of Tumours of Soft Tissue and Bone. 4th ed. Lyon: IARC, 2013.

2. Tabareau-Delalande F, Collin C, Gomez-Brouchet A, et al. Diagnostic value of investigating GNAS mutations in fibro-osseous lesions: a retrospective study of 91 cases of fibrous dysplasia and 40 other fibroosseous lesions. Mod Pathol 2013; 26: 911-21.

3. Salinas-Souza C, De Andrea C, Bihl M, et al. GNAS mutations are not detected in parosteal and low-grade central osteosarcomas. Mod Pathol 2015; 28: 1336-42.

4. Kanazawa I, Yamauchi M, Yano S, et al. Osteosarcoma in a pregnant patient with McCune-Albright syndrome. Bone 2009; 45: 603-8.

5. Sugiura Y, Kanda H, Motoi N, et al. Osteosarcoma arising in fibrous dysplasia, confirmed by mutational analysis of GNAS gene. Pathol Res Pract 2018; 214: 318-24.

6. Wagner VP, Carlos R, Romañach MJ, et al. Malignant transformation of craniomaxillofacial fibro-osseous lesions: a systematic review. J Oral Pathol Med 2019; 48: 441-50.

7. Cheng J, Wang Y, Yu H, et al. An epidemiological and clinical analysis of craniomaxillofacial fibrous dysplasia in a Chinese population. Orphanet J Rare Dis 2012; 7: 1-6.

8. Ruggieri P, Sim FH, Bond JR, et al. Malignancies in fibrous dysplasia. Cancer 1994; 73: 1411-24.

9. Tanner HC, Dahlin DC, Childs DS. Sarcoma complicating fibrous dysplasia. Probable role of radiation therapy. Oral Surg Oral Med Oral Pathol 1961; 14: 837-46.

10. Baumhoer D, Amary F, Flanagan AM. An update of molecular pathology of bone tumors. Lessons learned from investigating samples by nex generation sequencing. Genes Chromosomes Cancer 2019; 55: 88-99.

11. Whelan JS, Davis LE. Osteosarcoma, chondrosarcoma, and chordoma. J Clin Oncol 2018; 36: 188-93.

DOI: https://doi.org/10.1016/j.pathol.2020.08.027

\section{Two cases of parosteal osteosarcoma with unusual histological and molecular features}

\section{Sir,}

Osteosarcomas arising from the cortical surface of bone can be subdivided into parosteal, periosteal, and high grade surface osteosarcomas, with parosteal osteosarcomas being the most well differentiated. ${ }^{1}$ Clinically, parosteal osteosarcomas show female predominance and generally occur in the third decade of life, with the distal femur, proximal tibia, and proximal humerus being the common sites of origin. ${ }^{2}$ In general, they are low grade tumours with good prognosis. ${ }^{3}$ Radiologically, they appear as a lobulated mass with a broad base of attachment to the bone, and irregular mineralisation $^{1}$; a cleavage plane between the tumour and the bone is sometimes noted. ${ }^{4}$ On gross examination, they appear as ossified masses, which may have a cartilaginous cap; soft areas representing dedifferentiation may be present. ${ }^{1}$ Classically, they are described as having a biphasic histological appearance with parallel trabeculae of woven bone, with intervening spindle cells showing moderate atypia and collagenous stroma; this is the so called 'streamer pattern'. Occasionally, other histological patterns are encountered, which may resemble desmoplastic fibroma or fibrous dysplasia. ${ }^{1}$ Dedifferentiation (presence of high grade areas arising from a classic parosteal osteosarcoma) may occur, and is associated with a poorer prognosis. ${ }^{5}$ Molecular studies show parosteal osteosarcomas to be associated with amplification of Cyclin dependent kinase $4(C D K 4)$ and Mouse double minute 2 (MDM2), with $87-100 \%$ of cases positive for either (or both) of these markers by immunohistochemical staining in two studies, ${ }^{6,7}$ and amplification of MDM2 by fluorescent in situ hybridization (FISH) seen in $83 \%$ of cases examined in a 2012 work. $^{8}$ As such, cases which present without the classical histological pattern or without typical molecular changes may mimic benign lesions and present a diagnostic challenge. We present two cases of parosteal osteosarcoma with atypical histological features diagnosed recently in our institution, and expand the morphological spectrum of parosteal osteosarcoma.

The first case is that of a 21-year-old Chinese female, with no relevant past medical history, who presented with a 2 year history of posterior left knee pain, swelling, and limited range of motion. She had tried acupuncture to no effect, and underwent magnetic resonance imaging (MRI) in a neighbouring country, revealing a $3.1 \mathrm{~cm}$ lobulated mass apparently arising from the left posterior knee joint. She presented to our institution seeking a second opinion where plain radiographs were performed, demonstrating a bony lesion with circumferential sclerosis arising from the posterior aspect of the left lower femur, with ill-defined cortical margins and no association with any soft tissue mass. Radiological differential diagnoses considered were chondromyxoid tumour and parosteal osteosarcoma (Fig. 1A,B). Computed tomography scan of the leg revealed a $2.8 \mathrm{~cm}$ exophytic lesion at the posterior aspect of left lower femur with a rim of ossification. At this point the differential diagnoses considered by the clinical team included osteochondroma, parosteal osteosarcoma, chondromyxoid tumour, or chondrosarcoma. She underwent en bloc resection of the mass, and the post-operative 


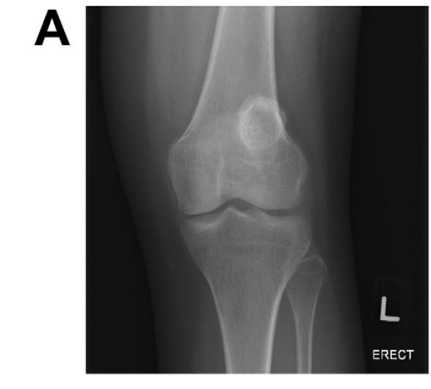

E

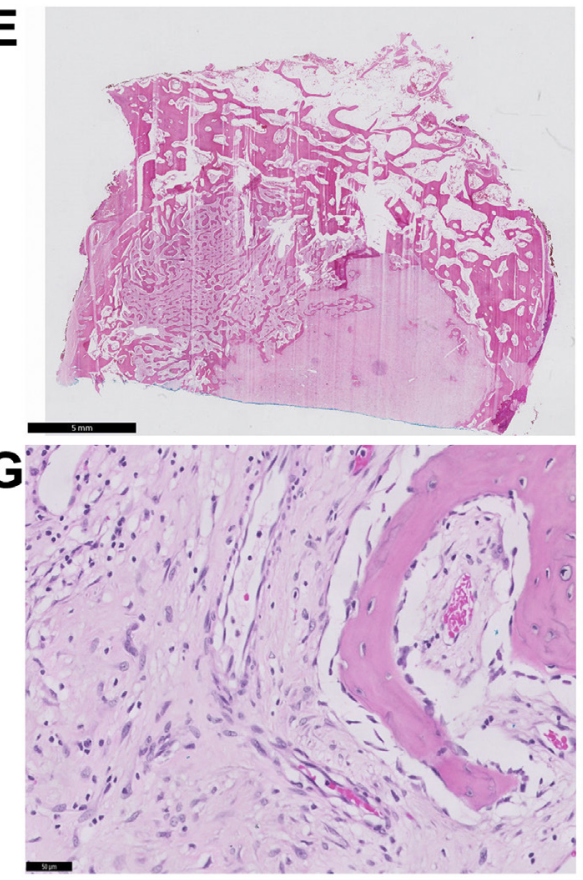

I

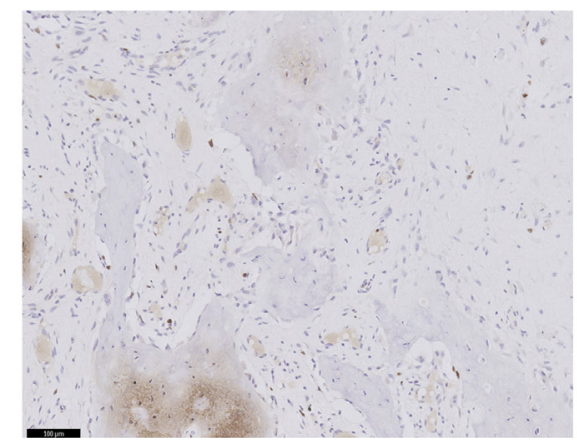

B

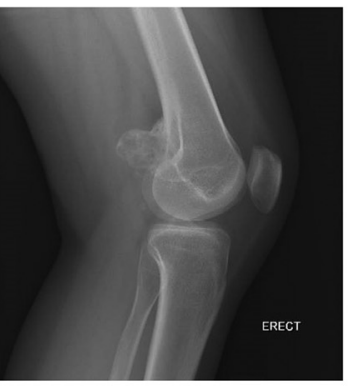

F

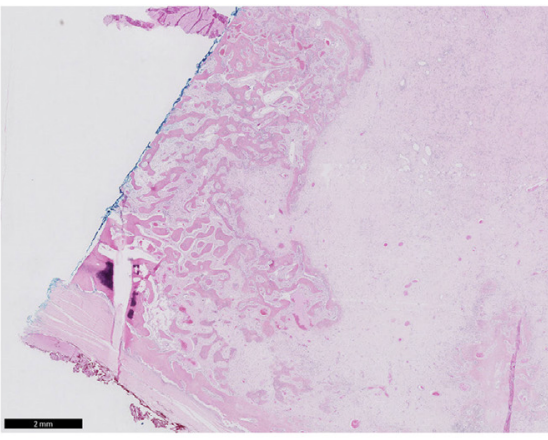

H

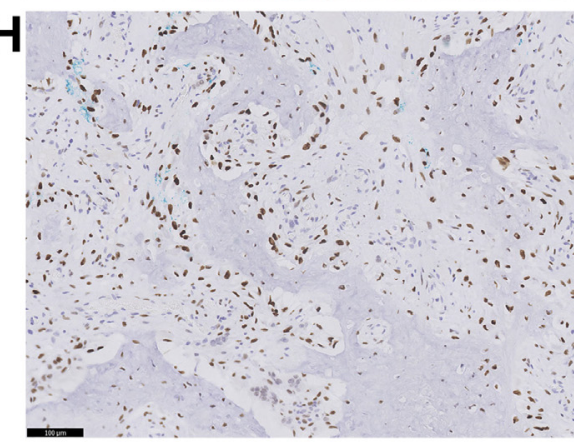

J

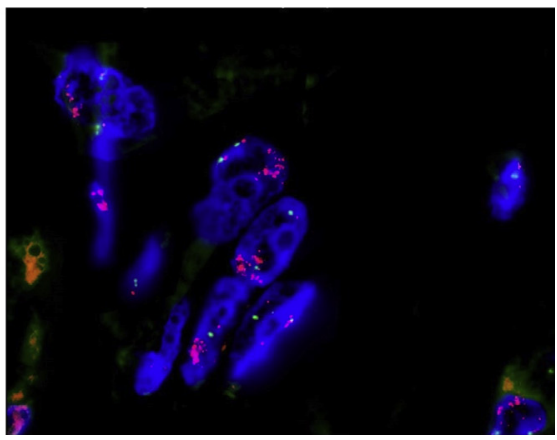

Fig. 1 Case 1. (A,B) Plain film radiographs of the left distal femur (A, antero-posterior view; B, lateral view), demonstrating a sclerotic bony lesion arising from the posterior aspect, with irregular cortical margins. (C) Gross pathological photograph of the resected mass demonstrating an irregular, rough, bony surface. (D) Gross pathological photograph of a representative cut section, demonstrating a rim of bony tissue, mixing with a central fibrous area. (E,F) Haematoxylin and eosin stained sections of the bony mass from Case 1 demonstrating a lesion consisting of a central spindle cell area, with ossification seen at the periphery, superficially resembling myositis ossificans. (G) Higher power view of the bony area of the lesion, demonstrating plump spindle cells with mild to moderate atypia in between trabeculae of woven bone. (H) SatB2 and (I) Ki-67 immunoperoxidase stained sections of the same lesion demonstrating focal positivity for SatB2 in the lesional cells, and low Ki-67 proliferative index. (J) Interphase FISH assay for MDM2 amplification (MDM2, red; CEP12, green) demonstrating increase in MDM2 copy number.

working diagnosis was osteochondroma. Examination of the resected specimen revealed a $3.6 \mathrm{~cm}$ bony mass, with firm, rough, bony tissue intermixed with softer, white, fibrous areas (Fig. 1C,D). Microscopic examination showed the centre of the lesion to resemble myositis ossificans and consist primarily of plump spindled to stellate cells with vesicular chromatin and small nucleoli, mild to moderate atypia, and occasional mitotic figures, set in abundant collagenous stroma (Fig. 1E-G). Irregular woven bone was noted primarily at the periphery of the lesion, and a rim of reactive bone was noted at the edge of the specimen (Fig. $1 \mathrm{E}-\mathrm{G}$ ). Nodules of hyaline cartilage were seen within the lesion. Based on the histomorphology, a diagnosis of myositis ossificans was first considered. Further immunohistochemical studies showed focal positivity for desmin, smooth muscle actin, and special AT-rich sequence binding protein2 (SATB2) in the lesional cells (Fig. 1H), with Ki-67 proliferative index of 5\% (Fig. 1I). Given the location and clinical concern for parosteal 
osteosarcoma, the specimen was sent for FISH studies, which showed amplification of MDM2 (Fig. 1J). Given the discrepancy between the morphological and molecular findings, the case was sent to an overseas expert for second opinion, and a final diagnosis of parosteal osteosarcoma, grade I, was made. The patient has had no evidence of recurrent disease 11 months after resection.

The second case is a 45 -year-old Chinese female, with a past medical history of hypertension, hyperlipidaemia, and benign uterine leiomyomata who presented to a general practitioner with a 3 month history of distal left humerus swelling. Clinical examination revealed a $3.0 \mathrm{~cm}$ bony swelling at the left distal humerus that was not tethered to the skin. Plain radiographs were obtained, which demonstrated a $2.5 \mathrm{~cm}$ osseous lesion at the posterolateral margin of the supracondylar region of the distal humerus (Fig. 2A,B). She was referred to our centre and MRI revealed a parosteal mass in the same location, with surrounding cortical and soft tissue oedema. The radiological differential diagnoses included florid reactive periostitis, bizarre periosteal
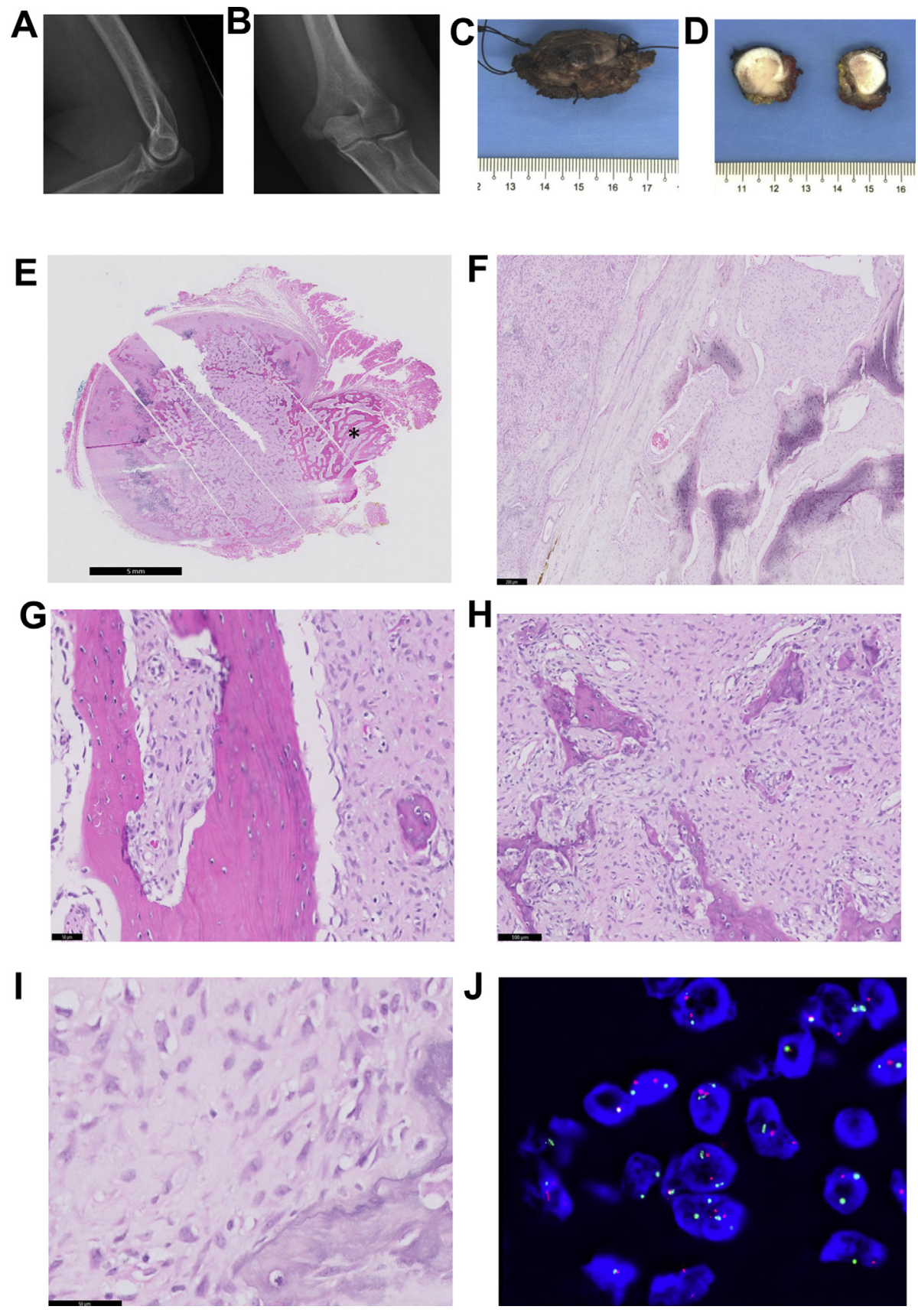

Fig. 2 Case 2. (A,B) Plain film radiographs of the left humerus (A, lateral view; B, antero-posterior view) demonstrating an exophytic juxtacortical osseous lesion arising from the supracondylar region of the left distal humerus. (C) Gross image of the resected mass demonstrating a firm, irregular, bony mass. (D) Gross image of a representative cut section, demonstrating a white, partially encapsulated bony nodule. (E-I) Haematoxylin and eosin stained sections of the bony mass from Case 2. (E) Low power overview of the lesion showing the exophytic mass and its relation to the native cortex $(*)$. (F) Lesional cells infiltrating the native cortex; $(\mathrm{G})$ higher magnification. (H) Centre of the lesion demonstrating spindle cells proliferating between short irregularly shaped blue to purple trabeculae of woven bone; (I) higher magnification. (J) Interphase FISH assay for MDM2 amplification (MDM2, red; CEP12, green) demonstrating no change in MDM2 copy number. 
osteochondromatous proliferation, myositis ossificans traumatic, periosteal osteosarcoma, and parosteal osteosarcoma. En bloc resection of the lesion with wide margins was performed. Gross examination of the specimen revealed a white, partially encapsulated bony nodule which measured $2.8 \mathrm{~cm}$ in maximum dimension (Fig. 2C,D). Microscopic examination revealed the lesion to have a nodular architecture with a surface composed of cartilage merging with underlying short, irregular trabeculae of blue to purple appearing woven bone, surrounded by plump spindle cells with oval nuclei, fine chromatin, and small nucleoli, showing mild atypia and occasional mitoses (Fig. 2E-I). These spindle cells infiltrated the cortex and percolated through the Haversian systems (Fig. 2E-G). Based on this histological appearance, the lesion was provisionally diagnosed as a bizarre parosteal osteochondromatous proliferation, but was sent for MDM2 amplification analysis by FISH, and for an external consultation. The FISH study showed no amplification of MDM2 (Fig. 2J), and immunohistochemical studies in the external consultant's laboratory showed focal positivity for $C D K 4$ and negativity for $M D M 2$. Despite the lack of $M D M 2$ amplification, and non-specific immunohistochemical profile, a final diagnosis of grade I parosteal osteosarcoma was made based on the cellular atypia, and the infiltrative growth pattern; the external consultant agreed with this interpretation. The patient has been well with no recurrence 29 months after resection.

Parosteal osteosarcoma is one of the more frequently misdiagnosed osteosarcomas, ${ }^{9}$ especially when the classic 'streamer' histological pattern is absent. It is unlikely to be misdiagnosed as a periosteal, or high grade surface osteosarcoma, as these higher grade malignancies show characteristic radiological patterns, and histologically would show much more marked atypia. ${ }^{1}$ However, if there is a dilemma between diagnosing a parosteal osteosarcoma with dedifferentiation and a conventional, high grade osteosarcoma, use of $M D M 2$ FISH can be useful, as MDM2 amplification tends to be retained in dedifferentiated parosteal osteosarcoma, and is not commonly seen in other high grade osteosarcomas. $8,10,11$ Confusion between parosteal osteosarcoma and other benign or reactive lesions is more likely, as they all represent lesions with only mild atypia. Both osteochondroma and parosteal osteosarcoma may show cartilaginous differentiation, but the cartilage seen in osteochondroma shows perpendicular columns of chondrocytes with intact polarity, with superficial clusters of chondrocytes in the superficial aspect, more mature chondrocytes at the base, and endochondral ossification. Furthermore, no interosseous spindle cell proliferation should be seen. Parosteal osteosarcoma may also be mistaken for myositis ossificans, as both lesions show myofibroblastlike cells intermixed with bony trabaculae; however, myositis ossificans should show a 'zonation' pattern with bony trabeculae appearing more mature at the periphery of the lesion. ${ }^{1}$ This was not observed in our first case, raising the suspicion that the lesion was in fact a parosteal osteosarcoma. Fibrous dysplasia is another mimic of parosteal osteosarcoma, as it may present as an exophytic mass and have similar histomorphological features, but fibrous dysplasia should show an intramedullary component. ${ }^{12}$ Finally, bizarre parosteal osteochondromatous proliferation (BPOP, Nora's lesion) may present as an exophytic bony lesion, which shows proliferating spindle cells, atypical-appearing chondrocytes, and endchondral ossification with 'blue' or 'purple' bony trabeculae, however, it is usually seen in the bones of the hands and the feet, and the spindle cells usually do not show nuclear hyperchromasia or cellular atypia. ${ }^{1}$ The presence of atypia, and infiltration of malignant cells into the cortex seen in the second case, raised the possibility that the lesion was actually a parosteal osteosarcoma. Examining CDK4 or MDM2 expression by immunohistochemistry, or alternatively, performing FISH or polymerase chain reaction (PCR) studies for $M D M 2$ amplification would be helpful to differentiate these benign mimics from parosteal osteosarcoma, as these markers appear to be quite specific for lowgrade osteosarcoma in this context. In one study immunohistochemical stains for CDK4 and MDM2 were performed on 23 low grade osteosarcomas and 40 benign mimics; all of the osteosarcomas showed expression of either or both of these markers, whereas only one case of a benign mimic (a bizarre parosteal osteochondromatous proliferation) showed weak staining for CDK4 and MDM2. ${ }^{6}$ In another study no expression of CDK4 and MDM2 was seen by immunohistochemistry in 107 benign fibro-osseous lesions but expression of either or both of these markers was seen in $89 \%$ of the low grade osteosarcomas studied. ${ }^{7}$ Furthermore, no amplification of MDM2 by PCR or FISH was seen in the benign fibro-osseous lesions that the authors examined. ${ }^{7}$ In our work, the positivity for MDM2 by FISH clinched the diagnosis in the first case, but the lack of positivity in the second required us to fall back to the morphology on routine haematoxylin and eosin stained sections for the final diagnosis.

Even though parosteal osteosarcoma is considered to be a low grade lesion with good clinical outcomes, there is a need to differentiate it from benign lesions. If wide margins are not taken, there are relatively high rates of local recurrence; with multiple recurrences, dedifferentiation can occur, which drastically negatively affects overall survival. ${ }^{3}$ Fortunately, with careful histological examination, and proper use of ancillary techniques, the proper diagnosis should be reached in most instances, sparing patients these negative outcomes.

Acknowledgements: The authors would like to thank Dr Andrew Rosenberg, University of Miami, Miller School of Medicine, for his review of the two cases presented herein.

Conflicts of interest and sources of funding: The authors state that there are no conflicts of interest to disclose.

\section{Benjamin Livingston Farah, Kesavan Sittampalam}

Department of Anatomical Pathology, Division of Pathology, Singapore General Hospital, Singapore

Contact Dr Kesavan Sittampalam.

E-mail: kesavan.sittampalam@singhealth.com.sg

1. Hang JF, Chen PCHC. Parosteal steosarcoma. Arch Pathol Lab Med 2014; 138: 694-9.

2. Okada K, Frassica FJ, Sim FH, et al. Parosteal osteosarcoma: a clinicopathological study. J Bone Joint Surg Am 1994; 76: 366-78.

3. Laitnen M, Parry M, Albergo JI, et al. The prognostic and therapeutic factors which influence the oncological outcome of parosteal osteosarcoma. Bone Joint J 2015; 97-B: 1698-703.

4. Jelinik JS, Murphey MD, Kransdorf MJ, et al. Parosteal osteosarcoma: value of MR imaging and $\mathrm{CT}$ in the prediction of histologic grade Radiology 1996; 201: 837-42. 
5. Ruengwanichayakun P, Gambarotti M, Frisoni T, et al. Parosteal osteosarcoma: a monocentric retrospective analysis of 195 patients. Hum Pathol 2019; 91: 11-8.

6. Yoshida A, Ushiku T, Motoi T, et al. Immunohistochemical analysis of MDM2 and CDK4 distinguishes low-grade osteosarcoma from benign mimics. Mod Pathol 2010; 23: 1279-88.

7. Dujardin F, Binh MB, Bouvier C, et al. MDM2 and CDK4 immunohistochemistry is a valuable tool in the differential diagnosis of lowgrade osteosarcomas and other primary fibro-osseous lesions of the bone. Mod Pathol 2011; 24: 624-37.

8. Duhamel LAE, Ye H, Halai D, et al. Frequency of mouse double minute 2 (MDM2) and mouse double minute 4 (MDM4) amplification in parosteal and conventional osteosarcoma subtypes. Histopathology 2012; 60: 357-9.

9. Grimer RJ, Carter SR, Spooner D, Sneath RS. Diagnosing musculoskeletal tumours Sarcoma 2001; 5: 89-94.

10. Yoshida A, Ushiku T, Motoi T, et al. MDM2 and CDK4 immunohistochemical coexpression in high-grade osteosarcoma: correlation with a dedifferentiated subtype. Am J Surg Pathol 2012; 36: 423-31.

11. Wunder JS, Eppert K, Burrow SR, et al. Co-amplification and overexpression of CDK4, SAS and MDM2 occurs frequently in human parosteal osteosarcomas. Oncogene 1999; 18: 783-8.

12. Dorfman HD, Ishida T, Tsuneyoshi M. Exophytic variant of fibrous dysplasia (fibrous dysplasia protuberans). Hum Pathol 1994; 25: 1234 -

DOI: https://doi.org/10.1016/j.pathol.2020.08.018

\section{A challenging case of a poorly differentiated small cell variant of synovial sarcoma with unusual immunophenotype}

Sir,

Diagnosis of a small cell variant of a poorly differentiated synovial sarcoma (PDSS) can be very challenging given the rarity of this subtype of synovial sarcoma ${ }^{1}$ and the very broad pathological differential diagnosis with other 'small blue round cell tumours', including various small round cell sarcomas, (small cell) carcinoma, lymphoblastic lymphoma and (small cell) melanoma. Recently, we have encountered a challenging case of a poorly differentiated small cell variant of a synovial sarcoma with an unusual immunophenotype.

A 35-year-old woman, without a history of trauma, presented with a soft tissue mass of the right subclavicular region. On clinical examination, the swelling was hard to palpate and measured approximately $6 \times 6 \mathrm{~cm}$. Magnetic resonance imaging (MRI) of the mass showed a lobulated, cystic soft tissue tumour with calcifications. After multidisciplinary discussion, an incision biopsy was performed. Microscopy showed a highly cellular, poorly differentiated malignant tumour composed of compact sheets of relatively uniform rounded cells with a high nuclear to cytoplasmic ratio (Fig. 1A). The cells had scant cytoplasm with indistinct borders ('small blue round cell' morphology) and contained vesicular nuclei with prominent nucleoli, demonstrating nuclear overlap (Fig. 1C). Brisk mitotic activity was observed (over 30 mitoses/10 high power fields). Very focally, an increase of myxoid stroma with ectatic (staghorn-like) vessels could be seen (Fig. 1B,D). Tumour necrosis and calcifications were absent. Immunohistochemistry showed diffuse, membranous staining for CD99 (Fig. 2A), together with strong nuclear transducing-like enhancer of split 1 (TLE1) reactivity of tumour cells (Fig. 2B). Patchy expression for epithelial membrane antigen (EMA) (Fig. 2C), cytokeratin AE1/AE3 (Fig. 2D), BCOR (Fig. 2E) and desmin (Fig. 2F) was observed. No aberrant immunoreactivity was seen for SOX10, S100, CD45, terminal deoxynucleotide transferase (TdT), myeloperoxidase (MPO), insulinoma associated protein 1 (INSM1), MyoD1, myogenin (MYF4), NKX2.2,

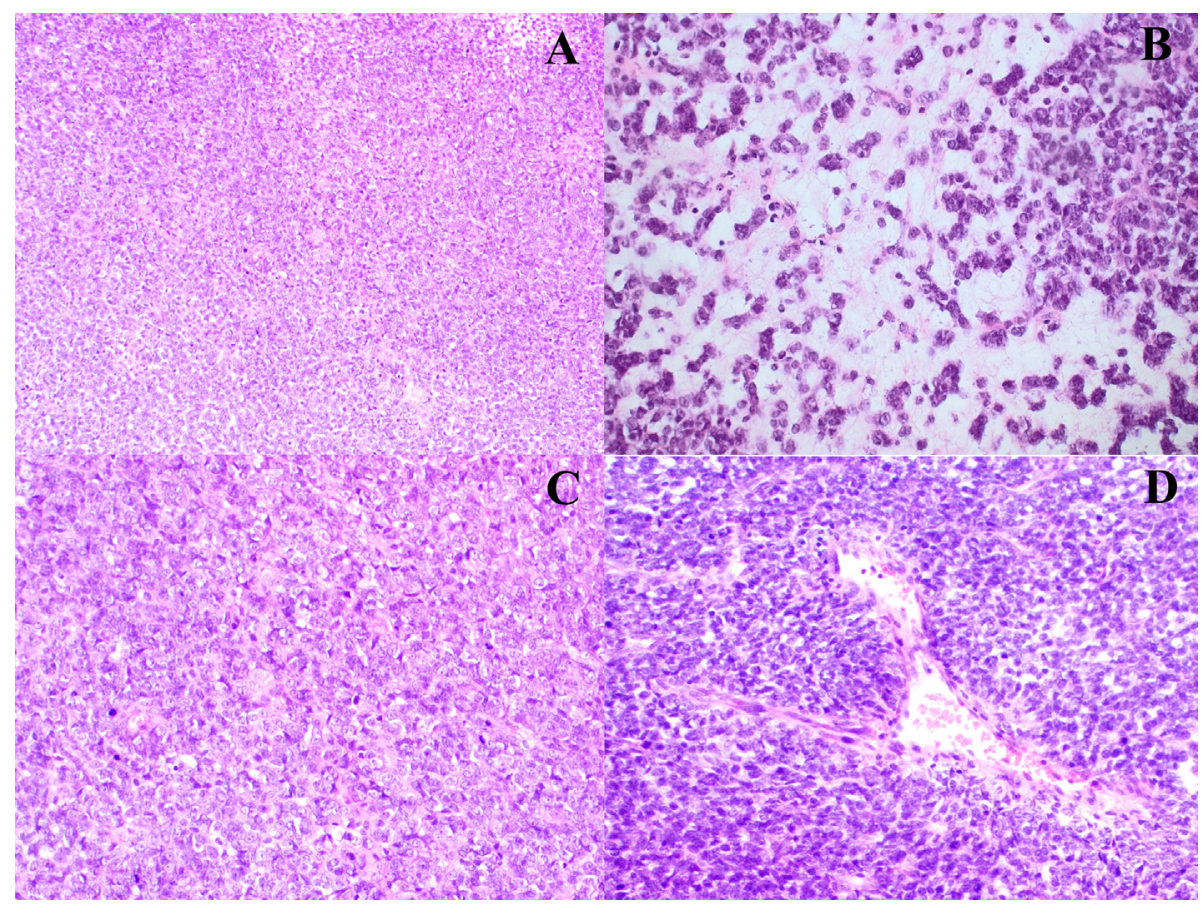

Fig. 1 (A) Highly cellular, malignant tumour composed of compact sheets of poorly differentiated rounded cells (H\&E). (B) Area with an increased myxoid stroma (H\&E). (C) Poorly differentiated rounded cells with a high nuclear to cytoplasmic ratio, vesicular nuclei with nucleoli and brisk mitotic activity (H\&E). (D) Presence of an ectatic (staghorn-like) vessel (H\&E). 